\title{
A REVIEW ON PROGRAM RECONSTRUCTING AND COMPONENT REUSE WITH CLUSTERING OF SOFTWARE COMPONENTS BASED ON FUZZY LOGIC
}

\author{
Huma Khan ${ }^{1}$, Yukti Kesharwani ${ }^{2}$, Rida Anwar ${ }^{3}$ \\ ${ }^{1}$ Research Scholar, Software Engineering, Institute Name, Chhattisgarh, INDIA \\ ${ }^{2}$ Assistant Professor, Computer Science, Institute Name, Chhattisgarh, INDIA \\ ${ }^{3}$ Research Scholar, Computer Science, Institute Name, Chhattisgarh, INDIA
}

\begin{abstract}
Delicate processing systems assume vital part in creating programming building applications. These comprise of fuzzy logic framework, neural system demonstrate and hereditary calculation procedures. Among these fuzzy logic and neural system procedures are comprehensively used to evaluate programming reusability, programming practicality, programming understandability and so on. Programming reuse is characterized as programming advancement with a few existing modules. In these a calculation to group an arrangement of given archives or content records or programming segments is planned which utilizes the proposed closeness work called half and half XNOR to discover the level of comparability among any two elements with fuzzy logic.
\end{abstract}

Keywords: Clustering, Fuzzy Logic, Software Reusability, Machine Training.

\section{INTRODCUTION}

Programming designing is one of the orders of software engineering, which gives superb programming, fulfills all predefined prerequisites and conveyed inside spending plan and time. Programming reusability is one of the quality characteristic in programming building, which signifies "the degree to which a product module or work item can be utilized again as a part of different applications with a specific end goal to incorporate new components". Modern specialists propose that around $20 \%$ of improvement expenses can be spared by utilizing reuse approach. Programming reuse diminishes the advancement time and cost.A product part essentially can't be separated from other programming components by the programming dialect used to execute the segment.

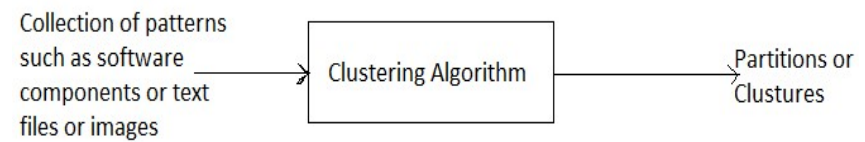

Fig. 1 abstract view of clustering process

The distinction must be in how programming segments are utilized. A product segment is a product component that complies with a part demonstrate and can be autonomously conveyed and created without change as indicated by a structure standard. A segment show characterizes particular collaboration and sythesis benchmarks. A segment show execution is the committed arrangement of executable programming components required to bolster the execution of parts that comply with the model. Discovering parts for productive programming reuse is one of the vital issues pointed by specialists. Grouping lessens the hunt space of segments by gathering comparable substances together in this manner guaranteeing lessened time many-sided quality as it diminishes the hunt time down segment recovery. Fuzzy logic, on the other hand, is a methodology that deals with uncertain and imprecise data and establishes the inputoutput relationship with the help of the fuzzy values and membership functions. Neural nets and fuzzy models are different to each other but still have close relationship. This paper is organized into following sections: related work, factors affecting software reusability, fuzzy logic and fuzzy model, implementation of fuzzy systems, artificial nueral network, machine training and learning functions, proposed fuzzy logic model, proposed nueral network model, discussion of results, conclusion and future prospects

\section{RELATED WORK}

An exceptionally expansive study by scientists, which thought of some as elements for the evaluation of programming reusability. The issue of discovering incessant itemsets is initially started from [9] which utilizes visit things to discover affiliation governs in extensive valuebased databases. In [2] grouping a given arrangement of content reports from neighbor set is proposed. In [3] the creators propose a strategy for finding most extreme length visit thing sets. In [7], the grouping of content records or reports is finished by considering Gaussian participation capacity and making utilization of it to acquire bunches by discovering word designs. Every group is recognized by its assertion design computed utilizing fluffy based Gaussian participation work once bunches are shaped. In this paper the thought is to first acquire visit thing sets for every record utilizing existing affiliation administer mining calculations either by even or vertical approach. When we find visit 
itemsets in every record then we shape a Boolean lattice with lines showing archives and sections demonstrating special successive things from every report. This is trailed by the calculation of a ternary element vector for every archive match, spoke to as a $2 \mathrm{D}$ exhibit or $2 \mathrm{D}$ grid by reclassifying the XNOR work as half and half XNOR rationale with slight change in the capacity presenting high impedance variable as $Z$. The possibility of greatest catching is taken as the base system for grouping [4]. The creators perform grouping utilizing XOR closeness work [15].

\section{PROBLEM IDENTIFICATION}

Reusability assumes an essential part in selecting a segment for programming frameworks. It helps in better understanding, enhance the proficiency for the framework and lower the endeavors and cost for the applications. Our exertion examined delicate figuring based systems i.e. Fluffy rationale and Neural Network to evaluate the Reusability of the part. Programming reusability is probably going to have a splendid future and a noteworthy work for research. This exertion will help engineers and scientist to pick the finest part identified with the reusability, which would help in enhancing the execution and proficiency of the entire programming system. Our point is to gauge high reusability of segments since it has been generally acknowledged in both scholarly community and industry for building reusable segments.

\section{PROPOSED METHODOLOGY}

Program rebuilding or code reusability can be utilized to change such projects or ineffectively composed ones to another frame that is better sorted out and simpler to comprehend, without changing the conduct of the projects. For that, the information set is a component attribute information lattice. Parts are the elements that we need to assemble in light of their likenesses. The parts could be programming modules; the traits, an arrangement of features. The Program rebuilding and segment reuse approach depends on bunching examination for the elements and their properties separated from source code. It additionally monitors programming upkeep cost. Taking after various grouping methods for part reuse and program rebuilding are-

\subsection{Hierarchical Agglomerative Clustering (HAC)}

To allot every part to a group we utilize HAC calculation. This calculation consolidates the two nearest comparable bunches. It will be rehashed until; there is stand out group left. Weighted Pair-Group Method using Arithmetic Averages (WPGMA), Complete Linkage Algorithm (CLINK), and Single Linkage Algorithm (SLINK) are the three generally utilized HAC calculations. The separation between two groups in WPGMA is finding by taking the normal of separation between all sets of parts in the two bunches. Clunk takes the separation between the most removed combine of segments, one from every bunch SLINK find by measuring the separations between the nearest match of segments, taking one segment from every group. There are numerous issue with HAC calculation that are when vast measure of information is given then its perform it gradually and it is exceptionally touchy with ending basis that is by errors it can consolidate profitable bunches into one group furthermore it not scale well. Already done things can never be fix by utilizing HAC calculation. It needs to recalculate on and on at every reiteration keeping in mind the end goal to discover comparability between groups.

\subsection{Fuzzy Logic}

Fuzzy logic is a strategy that arrangements with indeterminate and loose information. Fuzzy logic framework can be displayed with little information or with no information. Fuzzy logic system has numerous preferences over other delicate figuring methods. One of them is that it is less dependent on past qualities. It might likewise work without information. So it will bring about better comprehension the reuse undertaking. It considers elements like adaptability, cooperation multifaceted nature, comprehend capacity, shared trait and versatility as info while reusability of segment is considered as yield. This model can be utilized to anticipate the reusability of CBSD, which will help in evaluating improvement endeavors and quality for the application.

\subsection{Neural Network}

Neural Network (NN) can be utilized to speak to the complex non-direct affiliations (input-yield relationship) and capacities. NN is utilized to anticipate the reusability levels of programming by building up the relationship between the sources of info and yields in view of its preparation. It delivers the right yield from its preparation information or deficient information. NN model is more steady then the fluffy model for building the majority of the product and application. It is likewise used to speak to the product reusability usefulness.

\subsection{XNOR Similarity Function}

To plan a bunching calculation firstly we will need to make the similitude work which is the center part of any grouping calculation. For the most part we utilize Hybrid XNOR work as a comparability capacity, which is utilized to compute the comparative elements between any combine of substances which might be programming segments or reports. The records might be a product item report or a code sections or it might be content documents to be grouped. The contribution for parts grouping calculations are programming segments with properties predefines and the yield for segments bunching calculations is an arrangement of exceedingly durable segments with low coupling highlight. As indicated by the XNOR similitude work we discover the likeness between the two reports and it will create esteem $\mathrm{Z}$ (thinking pessimistically) when the element is nonappearance in one of the archives and it will deliver esteem zero when the element is nonattendance in both International Journal of records and it will deliver esteem one if the element is available in both the reports. It standardize the records profoundly strong programming part is create by the utilization this calculation. 


\subsection{Fuzzy Clustering}

One test in bunching is to recognize the group for a few segments that don't uncover solid association with a specific group or any bunches, or have high coupling with numerous bunches. It has been proposed to distinguish those "fluffy" segments by demonstrating express enrollment values that a part is related inside every single imminent group. The participation qualities are an indication of which bunch a segment has a place with. The part has a place with the bunch with which it has the most astounding participation esteem. Fluffy bunching has three necessities that is Membership esteem or degree, Total Membership, No void groups are permitted and no bunch may contain every one of the information. Contrasted and diverse bunching procedure, Fuzzy grouping is more adaptable and reasonable for true confounded uneven dispersed information, in light of the fact that amid the bunching emphasis, information cases can have a place with more than one group with various participation values. Fluffy bunching gets the ideal result.

\section{CONCLUSION}

Reusability assumes an essential part in selecting a segment for programming frameworks. It helps in better understanding, enhance the proficiency for the framework and lower the endeavors and cost for the applications. Our exertion examined delicate figuring based systems i.e. Fuzzy logic and Neural Network to evaluate the Reusability of the part. Programming reusability is probably going to have a splendid future and a noteworthy work for research. This exertion will help engineers and scientist to pick the finest part identified with the reusability, which would help in enhancing the execution and proficiency of the entire programming system. Our point is to gauge high reusability of segments since it has been generally acknowledged in both scholarly community and industry for building reusable segments.

\section{ACKNOWLEDGEMENT}

For this paper, a large amount of credit must go to our guide Mrs. Yukti Kesharwani. The author expresses sincere thanks to her for her continuous assistance, patience and support in the preparation of this paper.

\section{REFERENCES}

[1] Charu singh,AmrendraPratap and Abhishek Singhal. Estimation of sotware reusability for componentbased system using soft computing techniques, 2014 IEEE.

[2] Luo C, Li Y, Chung SM. Text document clustering based on neighbors, Data \& Knowledge Engineering, 2009; 68: 1271-1288.

[3] Tianming Hu,Sam Yuan Sung, Hui Xiong, Qian Fu. Discovery of maximum length frequent itemsets, Information Sciences, 2008; 178: 69- 87.

[4] Wen Zhanga,, Taketoshi Yoshida, Xijin Tang, Qing Wang. Text clustering using frequent itemsets, KnowledgeBased Systems, 2010; 23: 379-388

[5] Wen Zhanga,Taketoshi Yoshida, Xijin Tang. A comparative study of TF*IDF, LSI and multi-words for text classification. Expert Systems with Applications, 2011; 38: 2758-2765.

[6] Labatut V, Cherifi H. Accuracy Measures for the Comparison of Classifiers, ICIT 2011 The 5th International Conference on Information Technology.

[7] Jung-Yi Jiang et.al A Fuzzy Self-Constructing Feature Clustering Algorithm for Text Classification. IEEE Transactions on Knowledge and Data Engineering, 2011; 23(3).

[8] Hajdinjak M, Bauer A. Similarity Measures for Relational Databases, Informatica, 2009; 33: 143-149.

[9] Agrawal R, Imielinski T, Swami A. Mining association rules between sets of items in very large databases, Proceedings of the ACM SIGMOD Conference on Management of data, 1993: 207-216.

[10] Beil F, Ester M, Xu XW. Frequent term-based text clustering, in: Proceedings of the 8th ACM SIGKDD International Conference on Knowledge Discovery and Data Mining, 2002: 436-442.

[11] Radhakrishna V, Srinivas C, Guru Rao CV. High Performance Pattern Search algorithm using three sliding windows , International Journal of Computer Engineering and Technology, 2012; 3: 543-552.

[12] Susheela Devi V, Narasimha Murthy M. Text Book on Pattern Recognition. An Introduction. University Press.

[13] Kebir S, Seriai AD, Chardigny S. Comparing and Combining Genetic and Cluster Algorithms for Software Component Identification, in the Proceedings of the ACM Fifth International Conference on Computer Science and Software Engineering, 2012: 1-8.

[14] Veras RC, Meira SRL, Oliveira ALI, Melo BJM. Comparitive study of clustering techniques for the organisation of software repositories, in the proceedings of 19th IEEE International Conference on Tools with Artificial Intelligence.

[15] Radhakrishna V, Srinivas C, Guru Rao CV. Document Clustering Using Hybrid XOR Similarity Function for Efficient Software Component Reuse. Procedia Computer Science, 2013; (17): 121-128. 